\title{
Preoperative Inversed Albumin-to-Globulin Ratio Predicts Worse Oncologic Prognosis Following Curative Hepatectomy for Hepatocellular Carcinoma
}

This article was published in the following Dove Press journal:

Cancer Management and Research

Cheng-Cheng Zhang, ${ }^{1, *}$ ChengWu Zhang, ${ }^{2, *}$ Hao Xing, ${ }^{3, *}$ Yu Wang, 4 ,* Lei Liang, (ID) ${ }^{2,5, *}$ Yong-Kang Diao, ${ }^{2,5, *}$ Ting-Hao Chen, ${ }^{6}$ Wan Yee Lau, ${ }^{3,7}$ Ping Bie, ${ }^{8}$ Zhi-Yu Chen, ' Tian Yang ${ }^{1,3}$

'Department of Hepatobiliary Surgery, Southwest Hospital, Third Military Medical University (Army Medical University), Chongqing, China; ${ }^{2}$ Department of Hepatobiliary, Pancreatic and Minimal Invasive Surgery, Zhejiang Provincial People's Hospital, People's Hospital of Hangzhou Medical College, Zhejiang, China; ${ }^{3}$ Department of Hepatobiliary Surgery, Eastern Hepatobiliary Surgery Hospital, Second Military Medical University (Navy Medical University), Shanghai, China;

${ }^{4}$ Department of Hepatobiliary Surgery, Chongqing University Cancer Hospital, Chongqing, China; ${ }^{5}$ Key Laboratory of Tumor Molecular Diagnosis and Individualized Medicine of Zhejiang Province, Hangzhou,

China; ${ }^{6}$ Department of General Surgery, Ziyang First People's Hospital, Sichuan, China; ${ }^{7}$ Faculty of Medicine, The Chinese University of Hong Kong, Shatin, New Territories, Hong Kong SAR, China; ${ }^{8}$ Department of Hepatobiliary, The Third Affiliated Hospital, Chongqing Medical University, Chongqing, China

*These authors contributed equally to this work

Correspondence: Zhi-Yu Chen Department of Hepatobiliary Surgery, Southwest Hospital, Third Military Medical University (Army Medical University), No. 30, Gaotanyan Street, Chongqing 400038, China Email chenzhiyu_umn@I63.com

Tian Yang

Department of Hepatobiliary Surgery, Eastern Hepatobiliary Surgery Hospital, Second Military Medical University (Navy Medical University), No. 225, Changhai Road, Shanghai 200438, China

Email yangtian6666@hotmail.com
Background: A normal albumin-to-globulin ratio (NAGR) in serum is greater than 1 . Inversed albumin-to-globulin ratio (IAGR $<1$ ) indicates poor synthetic liver function or malnutrition. The aim of this study is to evaluate whether preoperative IAGR was associated with worse oncologic survival after hepatectomy for hepatocellular carcinoma (HCC).

Patients and Methods: Patients who underwent curative hepatectomy for HCC between 2009 and 2016 in four centers were divided into the IAGR and NAGR groups based on their preoperative levels, and their clinical characteristics and long-term survival outcomes were compared. Univariable and multivariable Cox regression analyses were performed to identify risk factors of overall survival (OS) and recurrence-free survival (RFS).

Results: Of 693 enrolled patients, 136 (19.6\%) were in the IAGR group. Their 5-year OS and RFS rates were $31.6 \%$ and $21.3 \%$, respectively, which were significantly worse than the NAGR group $(43.4 \%$ and $28.7 \%$, both $P<0.001)$. The area under the receiver operating characteristic curves in predicting 5-year OS and RFS using the albumin-to-globulin ratio were 0.68 and 0.67 , respectively, which were significantly higher than albumin $(0.60$ and 0.59), globulin (0.56 and 0.57), Child-Pugh grading (0.61 and 0.60), Model for End-Stage Liver Disease Score (0.59 and 0.58), and Albumin-Bilirubin grading (0.64 and 0.63). Multivariable analyses identified that preoperative IAGR was independently associated with worse OS (HR: 1.444, 95\% confidence interval (CI): $1.125-1.854, P=0.004$ ) and RFS (HR: $1.463,95 \%$ CI: $1.159-1.848, P=0.001$ ).

Conclusion: Preoperative IAGR was useful in predicting worse OS and RFS in patients who underwent curative hepatectomy for HCC.

Keywords: hepatocellular carcinoma, hepatectomy, albumin, globulin, survival, recurrence

\section{Introduction}

Hepatocellular carcinoma (HCC) has high morbidity and poor survival rates all over the world. ${ }^{1}$ Hepatectomy is the first-line treatment aiming at cure in selected patients. ${ }^{2,3}$ However, a high post-hepatectomy tumor recurrence rate limits the long-term prognosis. ${ }^{4,5}$ Most studies reported 5-year HCC recurrence rates of $60 \%$ to $70 \%$ after curative resection. ${ }^{5,6} \mathrm{~A}$ better understanding of perioperative risk factors associated with tumor recurrence and long-term survival helps in surgical decision-making, although whether adjuvant therapy can improve longterm oncological prognosis following hepatectomy for HCC is still debatable. 
Liver function tests used in clinical practice routinely include serum albumin, globulin, bilirubin and transaminases levels. ${ }^{7,8}$ A low serum level of albumin (normal range $35-50 \mathrm{~g} / \mathrm{L}$ ) reflects either poor nutritional status of the patient or poor synthetic function of the liver. A high level of globulin (normal range 20 35 g/L) indicates immune system overactivity, which is often found in patients with chronic inflammatory diseases such as chronic hepatitis. ${ }^{8}$ A surrogate index by combining albumin with globulin to form the albumin-to-globulin ratio is often used clinically to access liver function. ${ }^{9}$ The normal threshold value of the albumin-to-globulin ratio is 1.0 to 2.0. Underproduction of albumin or overproduction of globulin leads to decrease of the albumin-to-globulin ratio. An inversed albumin-to-globulin ratio (IAGR, $<1.0$ ) is often found in patients with severe inflammatory liver diseases or cirrhosis. ${ }^{10,11}$ Moreover, the albumin-toglobulin ratio has also been demonstrated to correlate with prognosis of patients with various diseases, especially with malignant tumors such as colorectal cancer, ${ }^{12-14}$ urothelial carcinoma, ${ }^{15-19}$ gastric cancer, ${ }^{20-23}$ and lung cancer. $^{24-26}$ The correlation between preoperative albumin-to-globulin ratio with long-term prognosis in patients treated with hepatectomy for HCC has only been reported in 3 single-center studies with small sample sizes. ${ }^{27-29}$ Furthermore, two of these studies only performed prognostic analyses on overall survival but not on recurrencefree survival. ${ }^{27,29}$

Based on a multicenter database with more than 10 years of follow-up, the present study aimed to identify whether preoperative albumin-to-globulin ratio independently predicted long-term overall survival and recurrencefree survival outcomes in patients with HCC after partial hepatectomy with curative-intent.

\section{Patients and Methods}

\section{Patient Enrollment}

Patients with HCC who underwent partial hepatectomy with curative intent from January 2009 to December 2016 at four Chinese medical centers (Southwest Hospital, Zhejiang Provincial People's Hospital, Eastern Hepatobiliary Surgery Hospital, and Chongqing University Cancer Hospital) were identified, and their clinical data were retrospectively analyzed. Curative hepatectomy was defined as complete resection of all microscopic and macroscopic HCC tumors with microscopically clear resection margins in the surgical specimens (R0 resection). The exclusion criteria included patients:
(1) less than 18 years old; (2) with chronic renal dysfunction which can lead to abnormalities in serum globulin; (3) with recurrent HCC, R1 (microscopically positive) or R2 resection (macroscopically positive); (4) who died within 30 days of operation; (5) lost to follow-up within 90 days of surgery; and (6) who had missing data on important prognostic variables. Informed consent was obtained from all enrolled patients to use their data for clinical research, either on hospital admission or during routine preoperative interviews. The study was conducted in accordance with the Declaration of Helsinki. The study was approved by the Institutional Review Board of the Eastern Hepatobiliary Surgery Hospital of Shanghai, China (No: EHBHKY2019-K-005) and individual consent for this retrospective analysis was waived.

\section{Clinicopathological and Operative Variables}

The following clinical variables were reviewed: age, sex, American Society of Anesthesiologists (ASA) score, hepatitis $\mathrm{B}$ virus (HBV) or hepatitis $\mathrm{C}$ virus (HCV) infection, cirrhosis, portal hypertension, preoperative hemoglobin, platelet, international normalized ratio (INR), creatinine, alpha-fetoprotein (AFP), Child-Pugh grading, Model for End-Stage Liver Disease (MELD) Score, and Albumin-Bilirubin (ALBI) grading; and liver function tests, which included bilirubin, alanine aminotransferase (ALT), aspartate transaminase (AST), albumin, and globulin levels. All laboratory tests were performed within one week prior to hepatectomy. Cirrhosis was confirmed by histopathological examination. Portal hypertension was defined when there was presence of esophageal varices or splenomegaly with a low platelet count $\left(<100 \times 10^{9} / \mathrm{L}\right)$. Pathological variables of HCC included maximum tumor size, tumor number, macrovascular or microvascular invasion, satellite nodules, tumor differentiation and tumor encapsulation. Operative variables included intraoperative blood loss, intraoperative blood transfusion, type of liver resection, and extent of hepatectomy. Major hepatectomy was defined as resection of three or more Couinaud liver segments, and minor hepatectomy as resection of fewer than three segments. Anatomical liver resections were defined by the Brisbane 2000 nomenclature of liver anatomy, ${ }^{30}$ while non-anatomical liver resection included wedge resection or limited resection.

\section{Follow-Up After Surgery}

Patients were regularly followed-up at each medical center after discharge from hospital using a standard protocol for 
surveillance of HCC recurrence. The protocol at each of the follow-up visits included a detailed history taking, physical examination, blood tests (liver function and serum AFP level) and radiological examinations using abdominal ultrasonography or magnetic resonance imaging (MRI) or contrast-enhanced computed tomography (CT). ${ }^{31}$ The follow-up visits were conducted once every two months for the first 6 months after surgery, then once every 3 months for the next 18 months, and then once every 6 months subsequently. When recurrence of $\mathrm{HCC}$ was suspected, CT/MRI, bone scanning or positron emission tomography-CT were performed as clinically indicated. Tumor recurrence was defined as new appearance of intrahepatic or extrahepatic tumor nodule (s) with typical imaging features consistent with HCC on contrast-enhanced CT or MRI, with or without a rise in serum AFP level. The treatment for patients with tumor recurrence included re-resection, liver transplantation, transcatheter arterial chemoembolization (TACE), local ablation, targeted drug therapy or supportive therapy.

\section{Study Endpoints}

The primary endpoint of the study was overall survival (OS) and the secondary endpoint was recurrence-free survival (RFS). OS was calculated from the date of hepatectomy to the date of death, or to the date of last follow-up. RFS was calculated from the date of hepatectomy to the date of diagnosis of tumor recurrence for patients with recurrence, or from the date of operation to the date of death or date of last follow-up for patients without recurrence. All patients were followed-up until death or loss to follow-up until the date this study was censored on December 31, 2019.

\section{Statistical Analysis}

The variables were exhibited using frequency and percentage for categorical covariates, and mean \pm standard deviation (SD) or median (range) for continuous covariates. The Pearson's chi-square test was applied for categorical covariates, while the Student $t$-test or Mann-Whitney U-test was adopted for continuous covariates. For laboratory results, the upper and lower limits of the normal values were used to divide patients into the normal or abnormal groups. Patients were divided into two groups using the frequently used $400 \mu \mathrm{g} / \mathrm{L}$ as the cut-off value for preoperative AFP level. The OS and RFS rates were compared between the IAGR and NAGR groups using the KaplanMeier method generated by the Log rank test. A receiver operating characteristic (ROC) analysis was performed to evaluate the power of prediction of 5-year OS and RFS, and the area under the receiver operating characteristic curve (AUROC) and the 95\% confidence intervals (CIs) were calculated. The AUROCs were compared among the laboratory variables and the clinical scores reflecting liver function (including Child-Pugh grading, MELD score and ALBI grading). Only significant variables with a $P$ value $<$ 0.1 on univariable analyses were put into multivariate analyses using the Cox proportional hazard model. Hazard ratios (HRs) and their 95\% confidence intervals (CIs) were estimated in the univariable and multivariable Cox regression analyses. Statistical analysis was performed using SPSS $^{\circledR}$ version 25.0 (IBM, Armonk, New York, USA). $P$ values were 2 -sided and a $P$ value $<$ 0.05 was considered as statistically significant.

\section{Results}

\section{Patient Characteristics}

With predetermined inclusion criteria, 693 patients were enrolled into this study (Figure 1). There were $610(88 \%)$ males and $83(12 \%)$ females. Using 1.0 as the cut-off value for preoperative albumin-to-globulin ratio taken within one week prior to surgery, patients were divided into the NAGR $(\mathrm{n}=557,80.4 \%)$ and IAGR $(\mathrm{n}=136,19.6 \%)$ groups (mean albumin-to-globulin ratio: 1.48 vs 0.89 , respectively). The patients' clinical characteristics and operative variables of these two groups are summarized in Table 1. Patients in the IAGR group, when compared with the NAGR group, were significantly older (mean: 53.2 vs 50.7 years), had higher percentages of female ( $19.1 \%$ vs $10.2 \%)$, cirrhosis $(79.4 \%$ vs $68.9 \%$ ) and portal hypertension (45.6\% vs 30.2\%), but had worse Child-Pugh grading (grade B: $11.3 \%$ vs $3.5 \%$ ), and larger tumors (mean: $6.7 \mathrm{~cm}$ vs $5.9 \mathrm{~cm}$ ). The laboratory parameters showed significant differences in most variables between the two groups, including hemoglobin, platelet, creatinine, INR, bilirubin, albumin, globulin, ALT, and AST. In addition, the IAGR group had significantly more intraoperative blood loss (median $400 \mathrm{~mL}$ vs $300 \mathrm{~mL}, \mathrm{P}<0.001$ ) and a higher percentage of patients receiving intraoperative blood transfusion $(26.5 \%$ vs $18.0 \%, \mathrm{P}=0.025)$ than the NAGR group.

\section{Long-Term Oncological Outcomes}

The long-term oncological outcomes between the IAGR and NAGR groups are shown in Table 2. The death rate in the IAGR group was significantly higher than the NAGR group $(73.5 \%$ vs $52.6 \%, P<0.001)$. However, the difference in recurrence was not significant $(64.7 \%$ vs $61.2 \%$, $P=0.453)$. The OS and RFS curves between the two 


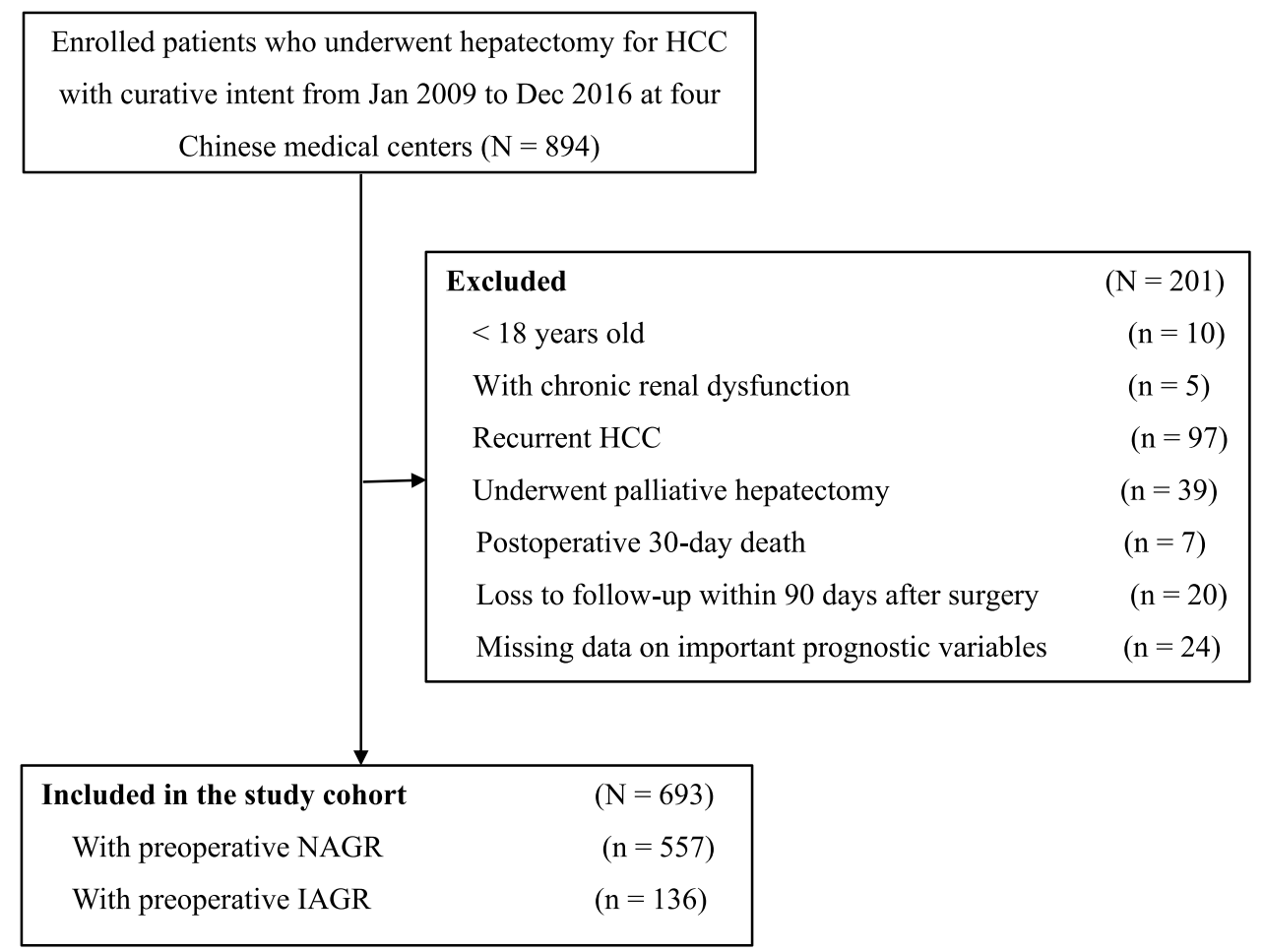

Figure I Selection of the study population.

Abbreviations: HCC, hepatocellular carcinoma; IAGR, inversed albumin-to-globulin ratio; NAGR, normal albumin-to-globulin ratio

groups are shown in Figure 2A and B. The median OS and RFS in the IAGR group were significantly worse in the NAGR group (50.8 vs 61.7 months, and 34.9 vs 45.4 months, both $P<0.001$ ). The 5-year OS and RFS rates in the IAGR group were $31.6 \%$ and $21.3 \%$, respectively, which were significantly worse than the NAGR group (43.4\% and $28.7 \%$, respectively, both $P<0.001$ ).

\section{ROC Analysis in Predicting 5-Year OS and RFS}

Table 3 shows the ROC analysis in the prediction of 5-year OS and RFS using the various laboratories and clinical variables. The AUROCs for 5-year OS and RFS using the albumin-toglobulin ratio were 0.68 and 0.67 , respectively, which were significantly better than the corresponding values using albumin only ( 0.60 and 0.59 ), globulin only ( 0.56 and 0.57 ), bilirubin only ( 0.55 and 0.55$)$, Child-Pugh grading $(0.61$ and 0.60 ), MELD score (0.59 and 0.58), and ALBI grading (0.64 and 0.63$)$. The best predictor for 5-year OS and RFS was the albumin-to-globulin ratio among all of these variables.

\section{Prognostic Analyses for OS and RFS}

The results on univariable and multivariable Cox regression analyses in predicting OS and RFS after curative hepatectomy for HCC are shown in Tables 4 and 5 . Multivariate Cox regression analyses demonstrated that IAGR was an independent risk factor in predicting worse OS (HR: 1.444, 95\% CI: 1.125-1.854, $P=0.004)$ and RFS (HR: $1.463,95 \%$ CI: $1.159-1.848$, $P=0.001)$.

\section{Discussion}

The present study was conducted to evaluate the relationship between albumin-to-globulin ratio with long-term survival outcomes after curative hepatectomy in patients with HCC. The albumin-to-globulin ratio combines both albumin and globulin levels to reflect the nutritional and inflammatory status of the patient. The commonly used normal value in clinical practice $(<1.0)$ was used to define an abnormal albumin-to-globulin ratio in this study. This threshold was then used to divide the enrolled patients into the IAGR and NAGR groups. Multivariable analyses revealed that IAGR was significantly associated with worse OS and RFS after curative hepatectomy for HCC. Hepatic surgeons should be alerted to the importance of preoperative albumin-to-globulin ratio in predicting longterm prognosis following $\mathrm{HCC}$ resection. In spite of the retrospective nature, the present study has the advantages 
Table I Comparisons of Clinicopathological and Operative Variables Between the IAGR and NAGR Groups

\begin{tabular}{|c|c|c|c|c|}
\hline Variables & $\begin{array}{l}\text { Total } \\
(\mathrm{N}=693)\end{array}$ & $\begin{array}{l}\text { IAGR Group } \\
(\mathrm{N}=136)\end{array}$ & $\begin{array}{l}\text { NAGR Group } \\
(\mathrm{N}=557)\end{array}$ & $P$ value \\
\hline Age, years* & $51.5 \pm 11.6$ & $53.2 \pm 10.4$ & $50.7 \pm 11.3$ & 0.001 \\
\hline Male sex & $610(88.0)$ & $110(80.9)$ & $500(89.8)$ & 0.004 \\
\hline ASA score $>2$ & $82(11.8)$ & $20(14.7)$ & $62(11.1)$ & 0.247 \\
\hline $\mathrm{HBV}(+)$ & $632(91.2)$ & $127(93.4)$ & $505(90.7)$ & 0.316 \\
\hline $\mathrm{HCV}(+)$ & $38(5.6)$ & $7(5.4)$ & $31(5.7)$ & $0.47 I$ \\
\hline Cirrhosis & $492(71.0)$ & $108(79.4)$ & $384(68.9)$ & 0.016 \\
\hline Portal hypertension & $230(33.2)$ & $62(45.6)$ & $168(30.2)$ & 0.001 \\
\hline Preoperative hemoglobin, $g / L^{*}$ & $142.1 \pm 17.3$ & $135.5 \pm 20.6$ & $144.2 \pm 16.9$ & $<0.001$ \\
\hline Preoperative platelet, $10^{9} / \mathrm{L}^{*}$ & $126.4 \pm 67.1$ & $121.5 \pm 68.3$ & $139.2 \pm 66.7$ & 0.001 \\
\hline Preoperative INR* & $1.09 \pm 0.12$ & $1.14 \pm 0.13$ & $1.08 \pm 0.11$ & $<0.001$ \\
\hline Preoperative creatinine, $\mu \mathrm{mol} / \mathrm{L}^{*}$ & $73.2 \pm 16.8$ & $69.4 \pm 14.6$ & $74.1 \pm 17.1$ & 0.003 \\
\hline Preoperative bilirubin, $\mu \mathrm{mol} / L^{*}$ & $15.0 \pm 5.8$ & $16.5 \pm 6.3$ & $14.6 \pm 5.6$ & 0.002 \\
\hline Preoperative albumin, $g / L^{*}$ & $40.9 \pm 4.0$ & $37.5 \pm 3.5$ & $41.8 \pm 3.7$ & $<0.001$ \\
\hline Preoperative globulin, g/L* & $30.4 \pm 5.1$ & $36.9 \pm 4.0$ & $28.7 \pm 3.9$ & $<0.001$ \\
\hline Preoperative albumin-to-globulin ratio* & $1.39 \pm 0.27$ & $0.89 \pm 0.09$ & $1.48 \pm 0.21$ & $<0.001$ \\
\hline Preoperative ALT, U/L* & $43.3(7.5-467.2)$ & $49.1(9.7-324.1)$ & $42.7(7.5-467.2)$ & 0.004 \\
\hline Preoperative AST, U/L* & $40.4(13.3-346.6)$ & $54.8(16.6-346.6)$ & $38.4(13.3-273)$ & $<0.001$ \\
\hline Child-Pugh grade, A/B & $631 / 62(91.8 / 8.2)$ & $120 / 16(88.7 / 11.3)$ & $537 / 20(96.5 / 3.5)$ & 0.049 \\
\hline MELD score* & $6.34 \pm 2.13$ & $7.18 \pm 2.31$ & $6.15 \pm 2.03$ & $<0.001$ \\
\hline ALBI grade* & $-2.7 \pm 0.36$ & $-2.4 \pm 0.33$ & $-2.8 \pm 0.32$ & $<0.001$ \\
\hline Preoperative AFP > $400 \mu \mathrm{g} / \mathrm{L}$ & $253(36.5)$ & $49(36.0)$ & $204(36.6)$ & 0.897 \\
\hline Maximum tumor size, $\mathrm{cm}^{*}$ & $6.0 \pm 3.9$ & $6.7 \pm 4.2$ & $5.9 \pm 3.8$ & 0.046 \\
\hline Multiple tumors & $153(22.1)$ & $28(20.6)$ & $125(22.4)$ & 0.640 \\
\hline Macrovascular invasion & $23(3.3)$ & $8(5.9)$ & $15(2.7)$ & 0.063 \\
\hline Microvascular invasion & $353(50.9)$ & $66(48.5)$ & $287(51.5)$ & 0.531 \\
\hline Satellite nodules & $162(23.4)$ & $31(22.8)$ & $|3|(23.5)$ & 0.858 \\
\hline Poor tumor differentiation & $55 \mid(79.5)$ & $107(78.7)$ & $444(79.7)$ & 0.788 \\
\hline Incomplete tumor encapsulation & $405(58.4)$ & $81(59.6)$ & $324(58.2)$ & 0.768 \\
\hline$B C L C$ staging, $A / B+C$ & $428 / 265(61.8 / 38.2)$ & $68 / 68(50 / 50)$ & $360 / 197(64.6 / 35.4)$ & 0.002 \\
\hline Intraoperative blood loss, mL* & $300(30-8000)$ & $400(50-8000)$ & $300(30-4600)$ & $<0.001$ \\
\hline Intraoperative blood transfusion & $136(19.6)$ & $36(26.5)$ & $100(18.0)$ & 0.025 \\
\hline Major hepatectomy & $165(23.8)$ & $31(22.8)$ & $134(24.1)$ & 0.756 \\
\hline Anatomical resection & 497 (7I.7) & $105(77.2)$ & $392(70.4)$ & 0.113 \\
\hline Resection margin $<\mathrm{I} \mathrm{cm}$ & $246(35.5)$ & $6 \mathrm{I}(44.9)$ & $185(33.2)$ & 0.011 \\
\hline
\end{tabular}

Note: *Values are mean \pm standard deviation or median (range) unless otherwise indicated.

Abbreviations: AFP, alpha-fetoprotein; ALBI, Albumin-Bilirubin; ALT, alanine aminotransferase; ASA, American Society of Anesthesiologists; AST, aspartate transaminase; BCLC, Barcelona Clinic Liver Cancer; HBV, hepatitis B virus; HCV, hepatitis C virus; IAGR, inversed albumin-to-globulin ratio; INR, international normalized ratio; MELD, Model for End-Stage Liver Disease; NAGR, normal albumin-to-globulin ratio.

of being a multicenter study using a large sample size with adequately long periods of follow-up, and carrying out analysis on multiple prognostic risk factors.

Serum albumin is commonly used in clinical practice as an indicator of liver function and nutritional status. It forms an important component in many clinical scores which are designed to reflect liver function, such as the Child-Pugh grading and the ALBI grading. ${ }^{32-34}$ When compared with albumin alone, the albumin-to-globulin ratio not only reflects liver function and nutritional status but also predicts the severity of chronic inflammation. ${ }^{9}$
As HCCs commonly have a background of chronic hepatitis $(91.2 \% \mathrm{HBV}$ infection and 5.6\% HCV infection in the present study), or even liver cirrhosis $(71.0 \%$ in the present study), and many studies have demonstrated that the severity of chronic hepatitis is associated with longterm survival outcomes and recurrence after HCC resection, ${ }^{35-38}$ the albumin-to-globulin ratio should be a more sensitive and specific test in predicting longterm prognosis than albumin only. In this study, the AUROCs in predicting 5-year OS and RFS were 0.68 and 0.67 using the albumin-to-globulin ratio, respectively, 
Table 2 Comparisons of Long-Term Oncologic Outcomes Between the IAGR and NAGR Groups

\begin{tabular}{|c|c|c|c|c|}
\hline N (\%) & $\begin{array}{l}\text { Total } \\
(\mathrm{N}=693)\end{array}$ & $\begin{array}{l}\text { IAGR Group } \\
(\mathrm{N}=136)\end{array}$ & $\begin{array}{l}\text { NAGR Group } \\
(\mathrm{N}=557)\end{array}$ & $P$ value \\
\hline Period of follow-up, months* & $47.6 \pm 35.5$ & $42.9 \pm 33.9$ & $49.2 \pm 35.9$ & 0.131 \\
\hline Death during the follow-up & $393(56.7)$ & $100(73.5)$ & $293(52.6)$ & $<0.001$ \\
\hline Recurrence during the follow-up & $429(61.9)$ & $88(64.7)$ & $34 \mid(6 I .2)$ & 0.453 \\
\hline \multicolumn{5}{|l|}{ Initial recurrence site } \\
\hline Intrahepatic only & $338(78.6)$ & $68(77.1)$ & $270(79.2)$ & 0.076 \\
\hline Extrahepatic only & $36(8.7)$ & $6(6.3)$ & $32(9.3)$ & \\
\hline Intrahepatic and Extrahepatic & $55(12.7)$ & $14(16.6)$ & 39 (II.5) & \\
\hline OS, months** & $59.6(56.9-62.2)$ & $50.8(44.8-56.7)$ & $61.7(58.8-64.6)$ & $<0.001$ \\
\hline I-year OS rate, \% & 93.9 & 89.7 & 95.0 & \\
\hline 3-year OS rate, \% & 71.6 & 60.3 & 74.3 & \\
\hline 5-year OS rate, \% & $4 I .1$ & 31.6 & 43.4 & \\
\hline RFS, months** & $43.3(40.7-45.9)$ & $34.9(29.3-40.5)$ & $45.4(42.5-48.3)$ & $<0.001$ \\
\hline I-year RFS rate, \% & 77.3 & 67.0 & 79.9 & \\
\hline 3-year RFS rate, \% & 53.0 & 41.2 & 55.8 & \\
\hline 5-year RFS rate, \% & 27.3 & 21.3 & 28.7 & \\
\hline
\end{tabular}

Notes: *Values are mean \pm standard deviation. $* *$ Values are median and $95 \%$ confidence interval.

Abbreviations: IAGR, inversed albumin-to-globulin ratio; NAGR, normal albumin-to-globulin ratio; OS, overall survival; RFS, recurrence-free survival.

which were significantly better than using albumin only (0.60 and 0.59). Furthermore, the albumin-to-globulin ratio predicted even significantly better than the commonly used clinical scoring systems, including the ChildPugh grading (0.61 and 0.60), the MELD scoring ( 0.59 and 0.58 , respectively), and the ALBI grading ( 0.64 and 0.63 , respectively). Thus, in patients with $\mathrm{HCC}$ with a background of chronic hepatitis, the performance of the albumin-to-globulin ratio in predicting prognosis after liver resection was the best among the existing liver functional scoring systems in the present study.

To avoid overlapping of variables on univariable and multivariable analyses of OS and RFS, only the albuminto-globulin ratio, bilirubin, ALT, and AST were put into the final model in the analysis. The following variables, including albumin, globulin, Child-Pugh grading, MELD scoring and ALBI grading (these scores contain albumin and/or bilirubin), were not put into the final model. The albumin-to-globulin ratio was shown to be an independent predictive factor of OS and RFS. Patients with resectable HCC but with an albumin-to-globulin ratio of less than 1.0 are predicted to have bad prognosis after surgery. If the surgical risk in these patients is high, or when hepatectomy is expected to be technically difficult, surgeons should be very cautious in recommending liver resection to such patients. A safer treatment modality in the form of local ablation or TACE should be considered.
In the present study, there were significant differences between the IAGR and NAGR groups of patients. The use of propensity score matching to examine the relationship between the preoperative albumin-to-globulin ratio and longterm oncologic outcomes for the purpose of balancing baseline characteristics before comparative analyses between the two groups may not be appropriate as this can lead to increase in selection biases between the two groups. As a consequence, the classical statistical approaches using univariable and multivariable Cox regression analyses were adopted in this study to find out whether IAGR was independently associated with worse OS and RFS following curative hepatectomy for $\mathrm{HCC}$, while adjusting for the other prognostic risk factors.

In addition to the albumin-to-globulin ratio, a number of other independent risk factors for worse OS and RFS were found in the present study. These risk factors included portal hypertension, preoperative AFP level $>400 \mu \mathrm{g} / \mathrm{L}$, maximum tumor size $>5.0 \mathrm{~cm}$, multiple tumors, macroscopic and microscopic vascular invasion, satellite nodules, and intraoperative blood transfusion. All these risk factors have been reported previously. ${ }^{31,37,39-41}$

The present study has limitations. First, HBV infection is the predominant etiology of HCC in this study from China, while the predominant etiology in most western countries and Japan is hepatitis $\mathrm{C}$ infection. Thus, the results from this study may not be applicable to HCC with other etiologies. Second, the retrospective study 

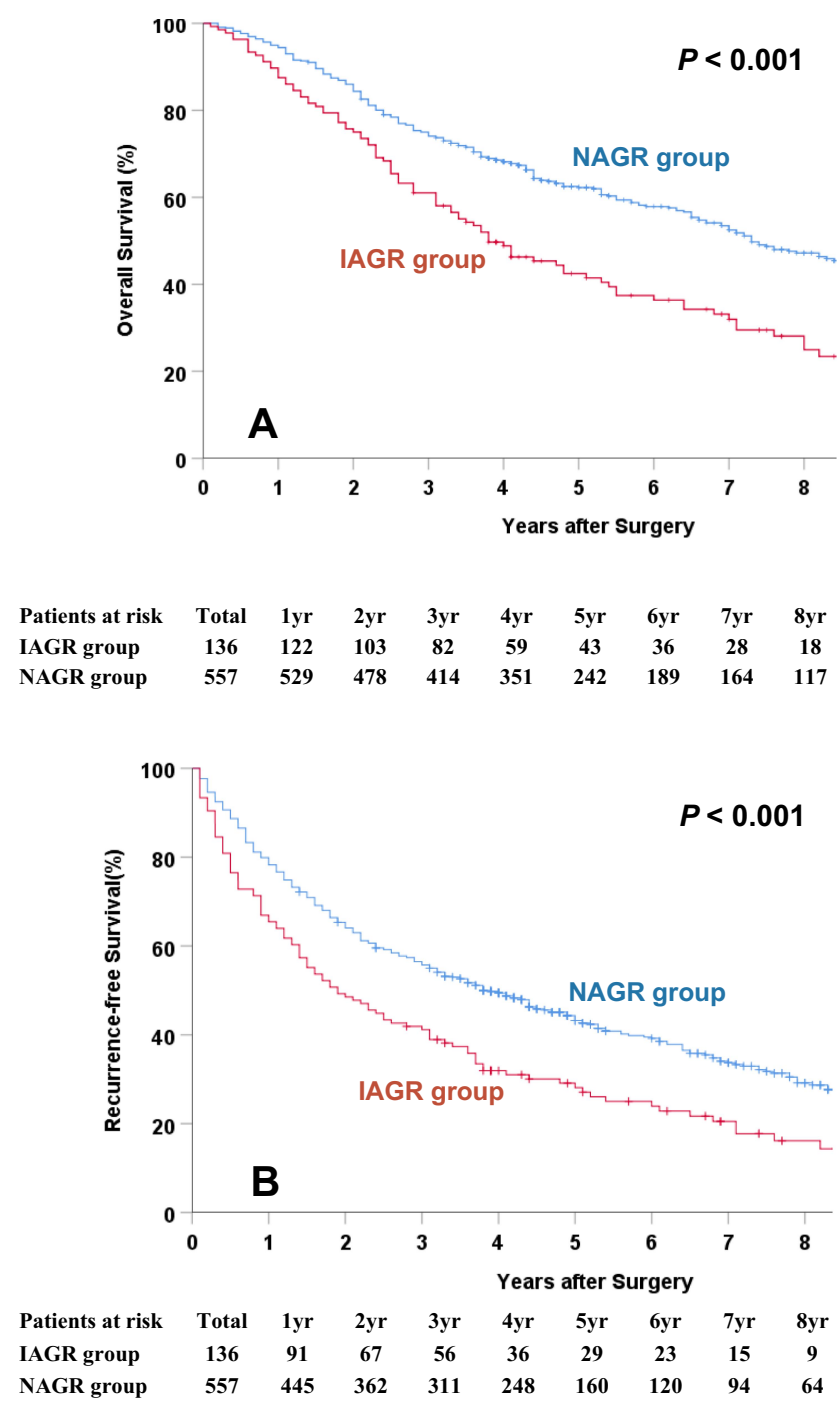

Figure 2 Overall survival (A) and recurrence-free survival (B) curves comparisons between the IAGR and NAGR groups. Abbreviations: IAGR, inversed albumin-to-globulin ratio; NAGR, normal albumin-to-globulin ratio.

with data collected from multi-centers has inherent defects. Third, some inflammatory and nutritional factors, such as neutrophil-lymphocyte ratio, platelet-lymphocyte ratio and sarcopenia, and other variables which can reflect liver functional reserves such as indocyanine green test and hepatic venous pressure gradient were not evaluated in the current study. ${ }^{42-47}$ For example, indocyanine green test has not always been carried out as a routine test in all participating hospitals as it did not cover by local Medicare in almost all Chinese provinces. More studies should be conducted in the future to incorporate these variables. Fourth, this study divided patients into the IAGR and NAGR groups by the commonly used value in clinical practice i.e. 1.0, as the cut-off value of the albumin-to-globulin ratio. Further studies should be carried out to identify the optimal threshold to separate the two groups of patients. In addition, further studies should be conducted to find out whether the albumin-toglobulin ratio can improve the commonly used Child-Pugh grading, MELD scoring and ALBI grading systems in assessing liver function, and in predicting outcomes after hepatectomy for HCC. Fifth, even indocyanine green (ICG) test was recognized as one of the most reliable prognostic factors for tolerance of hepatic surgery, routinely administrated in every patient especially in past years was not done. This variable was missing in characteristics in this study.

In conclusion, this multicenter study identified that preoperative IAGR was independently associated with worse OS and RFS following curative hepatectomy for HCC. This 
Table 3 Comparisons of ROC Analysis for the Prediction of Postoperative 5-Year Overall Survival and Recurrence-Free Survival

\begin{tabular}{|l|l|l|l|l|}
\hline $\begin{array}{l}\text { Preoperative Laboratory Indexes or Clinical Scores Reflecting } \\
\text { Liver Function }\end{array}$ & $\begin{array}{l}\text { AUROC for } \\
\mathbf{5 - Y e a r ~ O S}\end{array}$ & $\mathbf{9 5 \%} \mathbf{C l}$ & $\begin{array}{l}\text { AUROC for 5-Year } \\
\text { RFS }\end{array}$ & $\mathbf{9 5 \%}$ Cl \\
\hline Albumin & 0.60 & $0.54-0.64$ & 0.59 & \\
Globulin & 0.56 & $0.52-0.61$ & 0.57 & $0.55-0.63$ \\
Bilirubin & 0.55 & $0.52-0.60$ & 0.55 & \\
ALT & 0.54 & $0.52-0.58$ & 0.56 & $0.51-0.62$ \\
AST & 0.60 & $0.55-0.64$ & 0.61 & \\
Albumin-to-globulin ratio & 0.68 & $0.63-0.74$ & 0.67 & $0.51-0.59$ \\
Child-Pugh grade & 0.61 & $0.57-0.67$ & 0.60 & $0.56-0.65$ \\
MELD score & 0.59 & $0.52-0.63$ & 0.58 & $0.63-0.73$ \\
ALBI grade & 0.64 & $0.58-0.68$ & 0.63 & $0.57-0.64$ \\
\hline
\end{tabular}

Abbreviations: ALBI, Albumin-Bilirubin; ALT, alanine aminotransferase; AST, aspartate transaminase; AUROC, area under the receiver operating characteristic curve; Cl, confidence interval; MELD, Model for End-Stage Liver Disease; ROC, receiver operating characteristic; OS, overall survival; RFS, recurrence-free survival.

Table 4 Univariable and Multivariable Cox Regression Analyses in Predicting Overall Survival

\begin{tabular}{|c|c|c|c|c|c|}
\hline Variables & HR Comparison & UV HR (95\% Cl) & UV $P$ value & MV HR (95\% Cl) & MV P value * \\
\hline Age & $>60$ vs $\leq 60$ years & $0.897(0.698-1.152)$ & 0.395 & & \\
\hline Sex & Male vs Female & $1.054(0.777-1.430)$ & 0.395 & & \\
\hline ASA score & $>2$ vs $\leq 2$ & $0.967(0.7 \mid 4-1.309)$ & 0.829 & & \\
\hline HBV $(+)$ & Yes vs No & $1.376(0.937-2.022)$ & 0.104 & & \\
\hline $\mathrm{HCV}(+)$ & Yes vs No & $1.307(0.717-2.383)$ & 0.382 & & \\
\hline Cirrhosis & Yes vs No & $1.660(1.307-2.109)$ & $<0.001$ & NS & 0.213 \\
\hline Portal hypertension & Yes vs No & $1.408(1.148-1.726)$ & 0.001 & $1.296(1.108-1.701)$ & 0.041 \\
\hline Preoperative hemoglobin & $<110 \mathrm{vs} \geq 110 \mathrm{~g} / \mathrm{L}$ & $1.641(1.117-2.411)$ & 0.012 & NS & 0.909 \\
\hline Preoperative platelet & $<100$ vs $\geq 100 \times 10^{9} / \mathrm{L}$ & $1.217(1.042-1.326)$ & 0.009 & NS & 0.276 \\
\hline Preoperative bilirubin & $>17.1 \mathrm{vs} \leq 17.1 \mu \mathrm{mol} / \mathrm{L}$ & $1.359(1.105-1.673)$ & 0.004 & NS & 0.114 \\
\hline Preoperative albumin-to-globulin ratio & IAGR $(<1)$ vs NAGR $(\geq 1)$ & $1.736(1.383-2.181)$ & $<0.001$ & $1.444(1.125-1.854)$ & 0.004 \\
\hline Preoperative ALT & $>40$ vs $\leq 40 \mathrm{U} / \mathrm{L}$ & $1.279(1.045-1.565)$ & 0.017 & NS & 0.625 \\
\hline Preoperative AST & $>40 \mathrm{vs} \leq 40 \mathrm{U} / \mathrm{L}$ & $1.425(1.129-1.639)$ & $<0.001$ & NS & 0.109 \\
\hline Preoperative INR & $<1.17$ vs $\geq 1.17$ & $1.601(1.310-1.955)$ & 0.173 & & \\
\hline Preoperative creatinine & $>80 \mathrm{vs} \leq 80 \mu \mathrm{mol} / \mathrm{L}$ & $1.003(0.997-1.009)$ & 0.338 & & \\
\hline Preoperative AFP & $>400$ vs $\leq 400 \mu \mathrm{g} / \mathrm{L}$ & $1.659(1.356-2.028)$ & $<0.001$ & $1.311(1.058-1.624)$ & 0.013 \\
\hline Maximum tumor size & $>5.0 \mathrm{~cm}$ vs $\leq 5.0 \mathrm{~cm}$ & $2.015(1.650-2.462)$ & $<0.001$ & $1.464(1.148-1.867)$ & 0.002 \\
\hline Tumor number & Multiple vs Solitary & $2.122(1.694-2.656)$ & $<0.001$ & $1.151(0.804-1.649)$ & 0.441 \\
\hline Macrovascular invasion & Yes vs No & $2.388(1.468-3.886)$ & $<0.001$ & $2.761(1.860-3.925)$ & $<0.001$ \\
\hline Microvascular invasion & Yes vs No & $1.803(1.473-2.206)$ & $<0.001$ & $1.076(0.857-1.350)$ & 0.005 \\
\hline Satellite nodules & Yes vs No & $2.413(1.942-2.998)$ & $<0.001$ & NS & 0.528 \\
\hline Tumor differentiation & Poor vs Well/moderate & $1.769(1.352-2.314)$ & $<0.001$ & NS & 0.166 \\
\hline Tumor encapsulation & Incomplete vs Complete & $1.812(1.472-2.232)$ & $<0.001$ & NS & 0.193 \\
\hline Intraoperative blood loss & $>400 \mathrm{vs} \leq 400 \mathrm{~mL}$ & $1.588(1.276-1.903)$ & $<0.001$ & NS & 0.181 \\
\hline Intraoperative blood transfusion & Yes vs No & $1.842(1.463-2.319)$ & $<0.001$ & NS & 0.089 \\
\hline Extent of hepatectomy & Major vs Minor & $1.819(1.458-2.270)$ & $<0.001$ & NS & 0.435 \\
\hline Type of resection & Non-anatomical vs Anatomical & $0.883(0.703-1.107)$ & 0.280 & & \\
\hline Resection margin & $<\mathrm{I}$ vs $\geq \mathrm{I} \mathrm{cm}$ & $2.271(1.858-2.775)$ & $<0.001$ & NS & 0.153 \\
\hline
\end{tabular}

Note: *Those variables found significant at $P<0.1$ in univariable analyses were entered into multivariable Cox regression analyses.

Abbreviations: AFP, alpha-fetoprotein; ALT, alanine aminotransferase; ASA, American Society of Anesthesiologists; AST, aspartate transaminase; CI, confidence interval; HBV, hepatitis B virus; HCV, hepatitis C virus; HR, hazard ratio; IAGR, inversed albumin-to-globulin ratio; INR, international normalized ratio; MV, multivariable; NAGR, normal albumin-to-globulin ratio; NS, not significant; UV, univariable.

information allows hepatic surgeons to have a better preoperative prediction on the long-term oncologic prognosis for patients with HCC. The albumin-to-globulin ratio can be used as an easy, cheap, objective, and noninvasive biomarker for prognostication of patients with $\mathrm{HCC}$ undergoing curative hepatectomy. 
Table 5 Univariable and Multivariable Cox Regression Analyses in Predicting Recurrence-Free Survival

\begin{tabular}{|c|c|c|c|c|c|}
\hline Variables & HR Comparison & UV HR $(95 \% \mathrm{Cl})$ & UV $P$ value & MV HR $(95 \% \mathrm{Cl})$ & MV $P$ value * \\
\hline Age & $>60$ vs $\leq 60$ years & $0.803(0.633-1.019)$ & 0.071 & NS & 0.262 \\
\hline Sex & Male vs Female & $0.989(0.75 I-1.302)$ & 0.939 & & \\
\hline ASA score & $>2$ vs $\leq 2$ & $1.193(0.814-1.749)$ & 0.240 & & \\
\hline HBV $(+)$ & Yes vs No & $1.404(0.999-1.974)$ & 0.051 & NS & 0.378 \\
\hline $\mathrm{HCV}(+)$ & Yes vs No & $1.356(0.81 \mid-2.270)$ & 0.246 & & \\
\hline Cirrhosis & Yes vs No & $1.472(1.192-1.819)$ & $<0.001$ & NS & 0.252 \\
\hline Portal hypertension & Yes vs No & $1.211(1.004-1.460)$ & 0.045 & NS & 0.147 \\
\hline Preoperative hemoglobin & $<110$ vs $\geq 110 \mathrm{~g} / \mathrm{L}$ & $1.294(0.888-1.884)$ & 0.180 & & \\
\hline Preoperative platelet & $<100$ vs $\geq 100 \times 10^{9} / \mathrm{L}$ & $1.105(0.912-1.340)$ & 0.309 & & \\
\hline Preoperative bilirubin & $>17.1$ vs $\leq 17.1 \mu \mathrm{mol} / \mathrm{L}$ & $1.077(0.888-1.306)$ & 0.453 & & \\
\hline Preoperative albumin-to-globulin ratio & IAGR $(<1)$ vs NAGR $(\geq 1)$ & $1.574(1.271-1.950)$ & $<0.001$ & $1.463(1.159-1.848)$ & 0.001 \\
\hline Preoperative ALT & $>40$ vs $\leq 40 \mathrm{U} / \mathrm{L}$ & $1.317(1.096-1.582)$ & 0.003 & NS & 0.626 \\
\hline Preoperative AST & $>40$ vs $\leq 40 \mathrm{U} / \mathrm{L}$ & $1.168(1.348-1.941)$ & $<0.001$ & NS & 0.157 \\
\hline Preoperative INR & $<1.17$ vs $\geq 1.17$ & $1.279(1.104-1.395)$ & 0.361 & & \\
\hline Preoperative creatinine & $>80$ vs $\leq 80 \mu \mathrm{mol} / \mathrm{L}$ & $1.003(0.995-1.011)$ & 0.427 & & \\
\hline Preoperative AFP & $>400$ vs $\leq 400 \mu \mathrm{g} / \mathrm{L}$ & $1.528(1.270-1.839)$ & $<0.001$ & $1.239(1.021-1.504)$ & 0.030 \\
\hline Maximum tumor size & $>5.0 \mathrm{~cm}$ vs $\leq 5.0 \mathrm{~cm}$ & $1.924(1.603-2.308)$ & $<0.001$ & $1.373(1.106-1.704)$ & 0.004 \\
\hline Tumor number & Multiple vs Solitary & $1.851(1.503-2.279)$ & $<0.001$ & $1.292(1.103-1.681)$ & 0.036 \\
\hline Macrovascular invasion & Yes vs No & $3.420(2.199-5.320)$ & $<0.001$ & $2.548(1.610-4.033)$ & $<0.001$ \\
\hline Microvascular invasion & Yes vs No & $1.679(1.398-2.017)$ & $<0.001$ & $1.803(1.305-2.491)$ & 0.001 \\
\hline Satellite nodules & Yes vs No & $2.217(1.81 \mid-2.714)$ & $<0.001$ & $1.314(1.148-1.774)$ & 0.025 \\
\hline Tumor differentiation & Poor vs Well/moderate & $1.787(1.404-2.275)$ & $<0.001$ & NS & 0.626 \\
\hline Tumor encapsulation & Incomplete vs Complete & $1.795(1.486-2.167)$ & $<0.001$ & NS & 0.112 \\
\hline Intraoperative blood loss & $>400$ vs $\leq 400 \mathrm{~mL}$ & $1.413(1.177-1.696)$ & $<0.001$ & NS & 0.148 \\
\hline Intraoperative blood transfusion & Yes vs No & $1.680(1.355-2.082)$ & $<0.001$ & $1.515(1.132-2.207)$ & 0.012 \\
\hline Extent of hepatectomy & Major vs Minor & $1.829(1.493-2.242)$ & $<0.001$ & NS & 0.251 \\
\hline Type of resection & Non-anatomical vs Anatomical & $1.486(1.119-1.973)$ & 0.505 & & \\
\hline Resection margin & $<1$ vs $\geq 1 \mathrm{~cm}$ & $2.282(1.898-2.744)$ & $<0.001$ & NS & 0.261 \\
\hline
\end{tabular}

Note: *Those variables found significant at $P<0.1$ in univariable analyses were entered into multivariable Cox regression analyses.

Abbreviations: AFP, alpha-fetoprotein; ALT, alanine aminotransferase; ASA, American Society of Anesthesiologists; AST, aspartate transaminase; CI, confidence interval; HBV, hepatitis B virus; HCV, hepatitis C virus; HR, hazard ratio; IAGR, inversed albumin-to-globulin ratio; INR, international normalized ratio; MV, multivariable; NAGR, normal albumin-to-globulin ratio; NS, not significant; UV, univariable.

\section{Abbreviations}

AFP, alpha-fetoprotein; ALBI, Albumin-Bilirubin; ALT, alanine aminotransferase; AST, aspartate transaminase; ASA, American Society of Anesthesiologists; AUROC, area under the receiver operating characteristic curve; $\mathrm{CI}$, confidence interval; CT, computed tomography; HCC, hepatocellular carcinoma; HR, hazard ratio; HBV, hepatitis B virus; HCV, hepatitis $C$ virus; IAGR, albumin-to-globulin ratio; INR, international normalized ratio; MELD, Model for End-Stage Liver Disease; MRI, magnetic resonance imaging; NAGR, normal albumin-to-globulin ratio; NS, not significant OS, overall survival; SD, standard deviation; RFS, recurrence-free survival; ROC, receiver operating characteristic; TACE, transcatheter arterial chemoembolization.

\section{Author Contributions}

All authors made a significant contribution to the work reported, whether that is in the conception, study design, execution, acquisition of data, analysis and interpretation, or in all these areas; took part in drafting, revising or critically reviewing the article; gave final approval of the version to be published; have agreed on the journal to which the article has been submitted; and agree to be accountable for all aspects of the work.

\section{Funding}

New technique of laparoscope hepatobiliary surgery, 2010GXJ567 and Chinese National Key Projects, 201502014.

\section{Disclosure}

The authors report no conflicts of interest for this work.

\section{References}

1. Villanueva A. Hepatocellular carcinoma. $N$ Engl $J$ Med. 2019;380:1450-1462. doi:10.1056/NEJMra1713263 
2. Vibert E, Schwartz M, Olthoff KM. Advances in resection and transplantation for hepatocellular carcinoma. $J$ Hepatol. 2020;72:262-276. doi:10.1016/j.jhep.2019.11.017

3. Zhong JH, Torzilli G, Xing H, et al. Controversies and evidence of hepatic resection for hepatocellular carcinoma. BBA Clin. 2016;6:125-130. doi:10.1016/j.bbacli.2016.10.001

4. Maluccio M, Covey A. Recent progress in understanding, diagnosing, and treating hepatocellular carcinoma. CA Cancer $J$ Clin. 2012;62:394-399. doi:10.3322/caac.21161

5. Tung-Ping PR, Fan ST, Wong J. Risk factors, prevention, and management of postoperative recurrence after resection of hepatocellular carcinoma. Ann Surg. 2000;232:10-24. doi:10.1097/00000658200007000-00003

6. Poon RT. Prevention of recurrence after resection of hepatocellular carcinoma: a daunting challenge. Hepatology. 2011;54:757-759. doi:10.1002/hep.24569

7. Hoekstra LT, de Graaf W, Nibourg GA, et al. Physiological and biochemical basis of clinical liver function tests: a review. Ann Surg. 2013;257:27-36. doi:10.1097/SLA.0b013e31825d5d47

8. Agrawal S, Dhiman RK, Limdi JK. Evaluation of abnormal liver function tests. Postgrad Med J. 2016;92:223-234. doi:10.1136/postgradmedj-2015-133715

9. Knight JA. Liver function tests: their role in the diagnosis of hepatobiliary diseases. J Infus Nurs. 2005;28:108-117. doi:10.1097/ 00129804-200503000-00004

10. Tsochatzis EA, Bosch J, Burroughs AK. Liver cirrhosis. Lancet. 2014;383:1749-1761. doi:10.1016/S0140-6736(14)60121-5

11. Beardwood C, Gilbert C, Gillman J. Endocrine control of the plasma albumin/globulin ratio. Nature. 1962;195:710-711. doi:10.1038/ $195710 \mathrm{a} 0$

12. Azab B, Kedia S, Shah N, et al. The value of the pretreatment albumin/globulin ratio in predicting the long-term survival in colorectal cancer. Int J Colorectal Dis. 2013;28:1629-1636. doi:10.1007/ s00384-013-1748-z

13. Fujikawa H, Toiyama $Y$, Inoue $Y$, et al. Prognostic impact of preoperative albumin-to-globulin ratio in patients with colon cancer undergoing surgery with curative intent. Anticancer Res. 2017;37:1335-1342.

14. Xu Y, Xu X, Xi C, et al. Prognostic value of preoperative albumin to globulin ratio in elderly patients with rectal cancer. Medicine. 2019;98:e16066. doi:10.1097/MD.0000000000016066

15. Zhang B, Yu W, Zhou LQ, et al. Prognostic significance of preoperative albumin-globulin ratio in patients with upper tract urothelial carcinoma. PLoS One. 2015;10:e144961. doi:10.1371/journal. pone. 0144961

16. Liu J, Dai Y, Zhou F, et al. The prognostic role of preoperative serum albumin/globulin ratio in patients with bladder urothelial carcinoma undergoing radical cystectomy. Urol Oncol. 2016;34:484.e1484.e8. doi:10.1016/j.urolonc.2016.05.024

17. Liu Z, Huang H, Li S, et al. The prognostic value of preoperative serum albumin-globulin ratio for high-grade bladder urothelial carcinoma treated with radical cystectomy: A propensity score-matched analysis. J Cancer Res Ther. 2017;13:837-843. doi:10.4103/jcrt. JCRT_237_17

18. Fukushima H, Kobayashi M, Kawano K, et al. Prognostic value of albumin/globulin ratio in patients with upper tract urothelial carcinoma patients treated with radical nephroureterectomy. Anticancer Res. 2018;38:2329-2334.

19. $\mathrm{Xu} \mathrm{H}$, Tan $\mathrm{P}$, Ai J, et al. Prognostic impact of preoperative albumin-globulin ratio on oncologic outcomes in upper tract urothelial carcinoma treated with radical nephroureterectomy. Clin Genitourin Cancer. 2018;16:e10591059e1068. doi:10.1016/j.clgc.2018.06.003

20. Xue F, Lin F, Yin M, et al. Preoperative albumin/globulin ratio is a potential prognosis predicting biomarker in patients with resectable gastric cancer. Turk J Gastroenterol. 2017;28:439-445. doi:10.5152/ tjg.2017.17167
21. Toiyama Y, Yasuda H, Ohi M, et al. Clinical impact of preoperative albumin to globulin ratio in gastric cancer patients with curative intent. Am J Surg. 2017;213:120-126. doi:10.1016/j.amjsurg.2016.05.012

22. Mao MJ, Wei XL, Sheng H, et al. Clinical significance of preoperative albumin and globulin ratio in patients with gastric cancer undergoing treatment. Biomed Res Int. 2017;3083267.

23. Bozkaya Y, Erdem GU, Demirci NS, et al. Prognostic importance of the albumin to globulin ratio in metastatic gastric cancer patients. Curr Med Res Opin. 2019;35:275-282. doi:10.1080/ 03007995.2018.1479683

24. Yao Y, Zhao M, Yuan D, et al. Elevated pretreatment serum globulin albumin ratio predicts poor prognosis for advanced non-small cell lung cancer patients. J Thorac Dis. 2014;6:1261-1270.

25. Zhou T, He X, Fang W, et al. Pretreatment albumin/globulin ratio predicts the prognosis for small-cell lung cancer. Medicine. 2016;95: e3097. doi:10.1097/MD.0000000000003097

26. Wang $\mathrm{Y}, \mathrm{Li} \mathrm{S}, \mathrm{Hu} \mathrm{X}$, et al. The prognostic value of serum albumin-globulin ratio in early-stage non-small cell lung cancer: a retrospective study. Cancer Manag Res. 2019;11:3545-3554. doi:10.2147/CMAR.S191288

27. Deng Y, Pang Q, Miao RC, et al. Prognostic significance of pretreatment albumin/globulin ratio in patients with hepatocellular carcinoma. Onco Targets Ther. 2016;9:5317-5328. doi:10.2147/ OTT.S109736

28. Shimizu T, Ishizuka M, Suzuki T, et al. The preoperative globulin-toalbumin ratio, a novel inflammation-based prognostic system, predicts survival after potentially curative liver resection for patients with hepatocellular carcinoma. J Surg Oncol. 2017;116:1166-1175. doi: $10.1002 /$ jso. 24772

29. Zhang J, Liu X, Yang Z, et al. The pretreatment albumin to globulin ratio, a validated biomarker, predicts prognosis in hepatocellular carcinoma. J BUON. 2016;21:925-934.

30. Strasberg SM, Phillips C. Use and dissemination of the brisbane 2000 nomenclature of liver anatomy and resections. Ann Surg. 2013;257:377-382. doi:10.1097/SLA.0b013e31825a01f6

31. Xu XF, Xing H, Han J, et al. Risk factors, patterns, and outcomes of late recurrence after liver resection for hepatocellular carcinoma: a multicenter study from China. JAMA Surg. 2019;154:209-217. doi:10.1001/jamasurg.2018.4334

32. Spiekerman AM. Nutritional assessment (protein nutriture). Anal Chem. 1995;67:429R436R. doi:10.1021/ac00108a026

33. Pagliaro L. MELD: the end of Child-Pugh classification. J Hepatol. 2002;36:141-142. doi:10.1016/S0168-8278(01)00302-6

34. Johnson PJ, Berhane S, Kagebayashi C, et al. Assessment of liver function in patients with hepatocellular carcinoma: a new evidence-based approach-the ALBI grade. $J$ Clin Oncol. 2015;33:550-558. doi:10.1200/JCO.2014.57.9151

35. Franssen B, Alshebeeb K, Tabrizian P, et al. Differences in surgical outcomes between hepatitis B- and hepatitis C-related hepatocellular carcinoma: a retrospective analysis of a single north american center. Ann Surg. 2014;260:650-658.

36. Russell PS. Influence of accompanying chronic hepatitis status on recurrence of hepatocellular carcinoma after hepatectomy. Ann Surg. 1996;224:596-597. doi:10.1097/00000658-199611000-00002

37. Sasaki Y, Yamada T, Tanaka H, et al. Risk of recurrence in a long-term follow-up after surgery in 417 patients with hepatitis Bor hepatitis C-related hepatocellular carcinoma. Ann Surg. 2006;244:771-780. doi:10.1097/01.sla.0000225126.56483.b3

38. Chen VL, Le AK, Kim NG, et al. Effects of cirrhosis on short-term and long-term survival of patients with hepatitis b-related hepatocellular carcinoma. Clin Gastroenterol Hepatol. 2016;14:887-895.e1. doi:10.1016/j.cgh.2015.12.044

39. Poon RT, Fan ST, Lo CM, et al. Improving survival results after resection of hepatocellular carcinoma: a prospective study of 377 patients over 10 years. Ann Surg. 2001;234:63-70. doi:10.1097/ 00000658-200107000-00010 
40. Zheng J, Chou JF, Gönen M, et al. Prediction of hepatocellular carcinoma recurrence beyond milan criteria after resection: validation of a clinical risk score in an international cohort. Ann Surg. 2017;266:693-701. doi:10.1097/SLA.0000000000002360

41. Pinna AD, Yang T, Mazzaferro V, et al. Liver transplantation and hepatic resection can achieve cure for hepatocellular carcinoma. Ann Surg. 2018;268:868-875. doi:10.1097/ SLA.0000000000002889

42. Voron T, Tselikas L, Pietrasz D, et al. Sarcopenia impacts on shortand long-term results of hepatectomy for hepatocellular carcinoma. Ann Surg. 2015;261:1173-1183. doi:10.1097/SLA.000000000 0000743

43. Zhao Z, Liu J, Wang J, et al. Platelet-to-lymphocyte ratio (PLR) and neutrophil-to-lymphocyte ratio (NLR) are associated with chronic hepatitis $\mathrm{B}$ virus (HBV) infection. Int Immunopharmacol. 2017;51:1-8. doi:10.1016/j.intimp.2017.07.007
44. Ji F, Liang Y, Fu S, et al. Prognostic value of combined preoperative prognostic nutritional index and body mass index in HCC after hepatectomy. HPB (Oxford). 2017;19:695-705. doi:10.1016/j. hpb.2017.04.008

45. Pinato DJ, North BV, Sharma R. A novel, externally validated inflammation-based prognostic algorithm in hepatocellular carcinoma: the prognostic nutritional index (PNI). $\mathrm{Br} J$ Cancer. 2012;106:1439-1445. doi:10.1038/bjc.2012.92

46. Stremitzer S, Tamandl D, Kaczirek K, et al. Value of hepatic venous pressure gradient measurement before liver resection for hepatocellular carcinoma. Br J Surg. 2011;98:1752-1758. doi:10.1002/bjs.7672

47. Wang Y, Peng C, Cheng Z, et al. The prognostic significance of preoperative neutrophil-lymphocyte ratio in patients with hepatocellular carcinoma receiving hepatectomy: A systematic review and meta-analysis. Int J Surg. 2018;55:73-80. doi:10.1016/j.ijsu.2018.05.022

\section{Publish your work in this journal}

Cancer Management and Research is an international, peer-reviewed open access journal focusing on cancer research and the optimal use of preventative and integrated treatment interventions to achieve improved outcomes, enhanced survival and quality of life for the cancer patient.
The manuscript management system is completely online and includes a very quick and fair peer-review system, which is all easy to use. Visit http://www.dovepress.com/testimonials.php to read real quotes from published authors. 